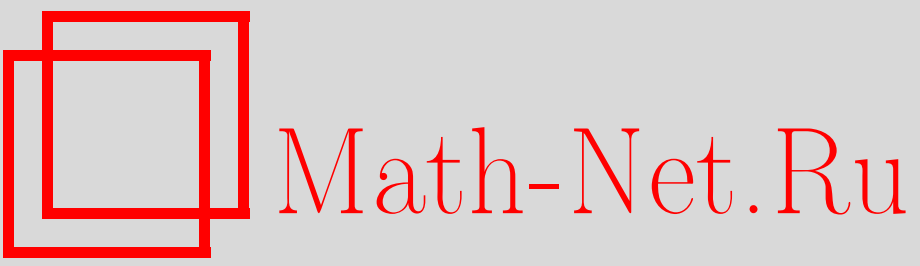

П. В. Парамонов, О $C^{m}$-продолжении субгармонических функций, Изв. РАН. Сер. матем., 2005, том 69, выпуск 6, 139-152

DOI: https://doi.org/10.4213/im670

Использование Общероссийского математического портала Math-Net.Ru подразумевает, что вы прочитали и согласны с пользовательским соглашением

http://www.mathnet.ru/rus/agreement

Параметры загрузки:

IP: 52.87 .193 .239

26 апреля 2023 г., 12:52:22 


\section{П.В. Парамонов}

\section{О $C^{m}$-продолжении субгармонических функций}

При $m \in(1,3)$ для любой (жордановой) $B$-области $D$ в $\mathbb{R}^{2}$ доказана возможность продолжения всякой субгармонической в $D$ функции класса $C^{m}(\bar{D})$ до функции, субгармонической и класса $C^{m}$ на всем $\mathbb{R}^{2}$ с оценкой $C^{m-1}$-нормы ее градиента. При $m \in[0,1) \cup[3,+\infty)$ аналогичное утверждение не верно даже для кругов. Указанные результаты остаются справедливыми для шаров $D$ в $\mathbb{R}^{N}$, $N \in\{3,4, \ldots\}$. Получен ряд следствий, а также соответствующие утверждения o Lip ${ }^{m}$-продолжении субгармонических функций.

Библиография: 7 наименований.

\section{§. Введение}

Пусть $D$ - жорданова область в $\mathbb{C}, B_{1}=\{w \in \mathbb{C}|| w \mid<1\}$ - единичный круг, $k: \overline{\mathbb{C}} \backslash \bar{D} \longrightarrow \overline{\mathbb{C}} \backslash \bar{B}_{1}-$ конформный изоморфизм с условиями $k(\infty)=\infty$ и $\lim _{z \rightarrow \infty}(k(z) / z)>0$ (он существует и определен однозначно по теореме Римана). Будем говорить, что $D$ является $B$-областью, если указанное отображение $k$ билипшицево, т. е. найдется константа $c_{1} \in[1,+\infty)$ такая, что

$$
c_{1}^{-1}\left|z_{1}-z_{2}\right| \leqslant\left|k\left(z_{1}\right)-k\left(z_{2}\right)\right| \leqslant c_{1}\left|z_{1}-z_{2}\right|
$$

при всех $z_{1}, z_{2} \in \mathbb{C} \backslash \bar{D}$. В частности, $\left|k^{\prime}(z)\right| \in\left[c_{1}^{-1}, c_{1}\right]$ для всех $z \in \mathbb{C} \backslash \bar{D}$. Отметим, что $\partial D$ не обязательно является $C^{1}$-гладкой кривой.

Для открытого множества $\Omega$ в $\mathbb{C}$ через $S H(\Omega)$ (соответственно, $H(\Omega)$ ) обозначается класс всех вещественнозначных субгармонических (соответственно, гармонических) функций в $\Omega$. Основной результат настоящей работы состоит в следующем.

Теорема 1.1. Пусть $D$ является $B$-областью, $m \in(1,3)$. Тогда найдется константа $c=c(D, m)>0$ такая, что для любой функции $f \in C^{m}(\bar{D}) \cap$ $S H(D)$ существует функиия $F \in C^{m}(\mathbb{C}) \cap S H(\mathbb{C})$ с условиями $\left.F\right|_{\bar{D}}=f u$

$$
\|\nabla F\|_{m-1, \mathbb{C}} \leqslant c\|\nabla f\|_{m-1, \bar{D}} .
$$

Далее, в $\S 2$, для всех $m \in[0,+\infty)$ вводятся (банаховы) пространства $B C^{m}(\Omega)$ (соответственно, $\left.C^{m}(X)\right)$ комплекснозначных " $m$-гладких" функций на открытом

Работа выполнена при частичной поддержке Российского фонда фундаментальных исследований (грант № 04-01-00720) и программы “Ведущие научные школы РФ" (грант НШ2040.2003.1). 
множестве $\Omega$ (соответственно, на компакте $X$ ) с нормами $\|f\|_{m, \Omega}$ (соответственно, $\left.\|f\|_{m, X}\right)$. Там же определяются пространства (Фреше) $C^{m}(\Omega)$.

Аналог теоремы 1.1 при $m=1$ справедлив при более жестких требованиях, налагаемых на область $D$ (см. [1, теоремы 2.2 и 3.1]). Как показывает следующее простое утверждение, случаи $m \in[0,1) \cup[3,+\infty)$ в рассматриваемом контексте особого интереса не представляют.

ПРЕДЛОЖЕНИЕ 1.2. При $m \in[0,1) \cup[3,+\infty)$ аналог теоремы 1.1 не верен дажсе для кругов в $\mathbb{C}$.

ДокаЗАТЕЛЬСтво. Действительно, пусть $m \in[0,1)$. Фиксируем $p \in(m, 1)$ и рассмотрим функцию $f(x)=-\left(1-|x|^{2}\right)^{p}$ в $B_{1}, f=0$ на $\partial B_{1}$, так что $f \in C^{m}\left(\bar{B}_{1}\right)$. Из соотношений

$$
\Delta f(x)=\left(1-|x|^{2}\right)^{p-2}\left(4 p-4 p^{2}|x|^{2}\right)>0
$$

в $B_{1}$ получаем, что $f \in S H\left(B_{1}\right)$, причем $\Delta f(x)$ не интегрируема в $B_{1}$ по мере Лебега. С другой стороны, как известно, всякая функция, субгармоническая в области $\Omega$, имеет в $\Omega$ обобщенный лапласиан, являюшийся локально конечной положительной борелевской мерой в $\Omega$. Поэтому $f$ нельзя продолжить (даже просто субгармонически) ни в какую окрестность компакта $\bar{B}_{1}$.

Пусть теперь $m \in[3,+\infty)$. Положим $f(x)=|x|^{2} / 4-|x|^{4} / 16, x \in \mathbb{R}^{2}$. Ввиду равенства $\Delta f(x)=1-|x|^{2}$ имеем

$$
\left.f\right|_{\bar{B}_{1}} \in C^{\infty}\left(\bar{B}_{1}\right) \cap S H\left(B_{1}\right)
$$

Далее, $\partial(\Delta f(x)) / \partial|x|=-2|x|<0$ на $\partial B_{1}$, поэтому при любом $C^{3}$-продолжении $F$ функции $f$ из $\bar{B}_{1}$ в какую-либо окрестность множества $\bar{B}_{1}$ мы будем иметь $\Delta F(x)<0$ для всех точек $x$ вне $\bar{B}_{1}$, достаточно близких к $\partial B_{1}$, а значит, $F$ не может быть субгармонической ни в какой окрестности замкнутого круга $\bar{B}_{1}$.

В $\S 2$ вводятся необходимые определения и обозначения.

Доказательство теоремы 1.1 приводится в $\S 3$.

В $\$ 4$ обсуждается ряд следствий: теорема о локализации (для произвольной жордановой области на плоскости), соответствуюшее (теореме 1.1) утверждение о Lip $^{m}$-продолжении субгармонических функций, а также аналогичные утверждения о $C^{m}$ - и $\operatorname{Lip}^{m}$-продолжении субгармонических функций с замкнутых шаров в $\mathbb{R}^{N}$.

В вопросе о точности условий теоремы 1.1 пока ясности нет. Автору известны примеры жордановых областей в $\mathbb{R}^{2}$ со спрямляемой гранищей, для которых утверждение теоремы 1.1 не имеет места.

\section{§2. Некоторые определения и обозначения}

Пусть $E$ - произвольное непустое множество в $\mathbb{C}, g$ - ограниченная комплекснозначная функция на $E$. Обозначим через $\|g\|_{E}$ равномерную норму $\sup _{x \in E}|g(x)|$ функции $g$ на $E$, а при $\mu \in[0,1]$ определим так называемый $\mu$-модуль непрерьвности функции $g$ на $E$ следуюшим образом:

$$
\omega_{E}^{\mu}(f, \delta)=\sup \frac{|g(x)-g(y)|}{|x-y|^{\mu}}, \quad \delta \in(0,+\infty]
$$


где указанный супремум (не обязательно конечный) берется по всем $x \in E$ и $y \in E$ таким, что $0<|x-y|<\delta$.

Всюду далее $x=\left(x_{1}, x_{2}\right) \in \mathbb{R}^{2}, z=x_{1}+i x_{2} \in \mathbb{C}$. Для открытого множества $\Omega$ в $\mathbb{C}$ и $m \in \mathbb{Z}_{+}=\{0,1, \ldots\}$ через $B C^{m}(\Omega)$ обозначается класс всех (комплекснозначных) функций $f$, имеющих непрерывные и ограниченные частные производные (по переменным $\left.x_{1}, x_{2}\right)$ в $\Omega$ до порядка $m$ включительно. Норма (банахова) в $B C^{m}(\Omega)$ задается следующим образом:

$$
\|f\|_{m, \Omega}=\max _{\{|\beta| \leqslant m\}}\left\|\partial^{\beta} f\right\|_{\Omega}
$$

где указанный максимум берется по всем 2 -индексам $\beta=\left(\beta_{1}, \beta_{2}\right) \in \mathbb{Z}_{+}^{2}$ с условием $|\beta|=\beta_{1}+\beta_{2} \leqslant m$, причем

$$
\partial^{\beta} f(x)=\frac{\partial^{|\beta|} f(x)}{\partial x_{1}^{\beta_{1}} \partial x_{2}^{\beta_{2}}} .
$$

При $m \in \mathbb{Z}_{+}$и $0<\mu \leqslant 1$ положим

$$
B \operatorname{Lip}^{m+\mu}(\Omega)=\left\{f \in B C^{m}(\Omega) \mid \omega_{\Omega}^{m, \mu}(f, \infty)<\infty\right\},
$$

где

$$
\omega_{E}^{m, \mu}(f, \delta)=\max \left\{\omega_{E}^{\mu}\left(\partial^{\alpha} f, \delta\right)\right\}, \quad E \subset \Omega, \quad \delta>0,
$$

а последний максимум берется по всем 2-индексам $\alpha$ с условием $|\alpha|=m$.

При $m \in \mathbb{Z}_{+}$и $0<\mu<1$ определим

$$
B C^{m+\mu}(\Omega)=\left\{f \in B \operatorname{Lip}^{m+\mu}(\Omega) \mid \omega_{K}^{m, \mu}(f, \delta) \rightarrow 0 \text { при } \delta \rightarrow 0\right\}
$$

(последнее условие выполнено для любого компакта $K$ в $\Omega$ ).

Норма в $B \operatorname{Lip}^{m+\mu}(\Omega)$ (при $\left.\mu \in(0,1]\right)$ и в $B C^{m+\mu}(\Omega)$ (при $\left.\mu \in(0,1)\right)$ определяется по формуле

$$
\|f\|_{m+\mu, \Omega}=\max \left\{\|f\|_{m, \Omega}, \omega_{\Omega}^{m, \mu}(f, \infty)\right\} .
$$

Для произвольного $m \geqslant 0$ и открытого множества $\Omega$ через $C^{m}(\Omega)$ (соответственно, $\operatorname{Lip}^{m}(\Omega), m>0$ ) обозначается класс всех функций $f$ в $\Omega$ таких, что $f \in B C^{m}(E)$ (соответственно, $\left.f \in B \operatorname{Lip}^{m}(E)\right)$ для любого ограниченного открытого множества $E$ с условием $\bar{E} \subset \Omega$. Ясно, что $C^{m_{1}}(\Omega) \subset C^{m_{2}}(\Omega)$ (и $\left.\operatorname{Lip}^{m_{1}}(\Omega) \subset \operatorname{Lip}^{m_{2}}(\Omega)\right)$ для любых $m_{1} \geqslant m_{2} \geqslant 0$. Определим еще $C^{\infty}(\Omega)=$ $\bigcap_{m \geqslant 0} C^{m}(\Omega)$. Пространства $C^{m}(\Omega)$ и $\operatorname{Lip}^{m}(\Omega)$ стандартным образом наделяются топологиями фреше. Нетрудно показать (для всех $m>0$ ), что $B C^{m}(\Omega)$ есть замыкание в $B \operatorname{Lip}^{m}(\Omega)$ подпространства $C^{\infty}(\Omega) \cap B \operatorname{Lip}^{m}(\Omega)$. Таким образом, классы $B C^{m}(\Omega)$ (и ниже классы $C^{m}(X)$ ) естественно возникают, например, в задачах аппроксимачии гармоническими функииями в нормах $\|\cdot\|_{m}$.

Мы не будем выписывать индексы $m$ и (или) $\Omega$ в обозначениях введенных норм при $m=0$ и (или) $\Omega=\mathbb{C}$.

Пусть теперь $X-$ компактное множество в $\mathbb{C}, m \geqslant 0$ произвольно. Через $C^{m}(X)$ (соответственно, $\operatorname{Lip}^{m}(X), m>0$ ) обозначим класс функций $f$ на $X$, 
которые допускают продолжения до функций класса $B C^{m}(\mathbb{C})$ (соответственно, $\left.B \operatorname{Lip}^{m}(\mathbb{C})\right)$. Для $f \in C^{m}(X)\left(\right.$ соответственно, $\left.f \in \operatorname{Lip}^{m}(X)\right)$ определим

$$
\|f\|_{m, X}=\inf \|F\|_{m}
$$

где указанный инфимум берется по всем возможным продолжениям $F \in B C^{m}(\mathbb{C})$ (соответственно, $\left.F \in B \operatorname{Lip}^{m}(\mathbb{C})\right),\left.F\right|_{X}=f$.

Отметим, что если внутренность $X^{\circ}$ компакта $X$ плотна в $X$ (т. е. $\overline{X^{\circ}}=X$, где $\bar{E}$-замыкание множества $E$ в $\mathbb{C})$, то для всякой $f \in C^{m}(X)$ однозначно определены функции $\partial^{\beta} f(x)$ на $X,|\beta| \leqslant[m]$. В частности, при $m \geqslant 1$ корректно определен вектор-градиент $\nabla f(x)=\left\{\frac{\partial f(x)}{\partial x_{1}}, \frac{\partial f(x)}{\partial x_{2}}\right\}$ на $X$ с модулем

$$
|\nabla f(x)|=\max _{\{1 \leqslant n \leqslant 2\}}\left|\frac{\partial f(x)}{\partial x_{n}}\right|
$$

при $m \geqslant 2$ матрица вторых частных производных

$$
\nabla^{2} f(x)=\left(\frac{\partial^{2} f}{\partial x_{n} \partial x_{n^{\prime}}}\right)_{n, n^{\prime} \in\{1,2\}}
$$

корректно определена на $X$, причем

$$
\left|\nabla^{2} f(x)\right|=\max _{\left\{1 \leqslant n \leqslant n^{\prime} \leqslant 2\right\}}\left|\frac{\partial^{2} f(x)}{\partial x_{n} \partial x_{n^{\prime}}}\right| .
$$

Если $Е$ открыто или компактно (причем в последнем случае потребуем, чтобы $\left.\overline{E^{\circ}}=E\right)$ и $f \in C^{m}(E)$, то положим

$$
\begin{aligned}
\|\nabla f\|_{m-1, E} & =\max _{\{1 \leqslant n \leqslant 2\}}\left\|\frac{\partial f}{\partial x_{n}}\right\|_{m-1, E} \text { при } m \geqslant 1, \\
\left\|\nabla^{2} f\right\|_{m-2, E} & =\max _{\left\{1 \leqslant n \leqslant n^{\prime} \leqslant 2\right\}}\left\|\frac{\partial^{2} f}{\partial x_{n} \partial x_{n^{\prime}}}\right\|_{m-2, E} \text { при } m \geqslant 2
\end{aligned}
$$

при условии, что эти полунормы конечны (последнее замечание касается только открытых множеств $E)$.

Общая постановка интересующей нас задачи состоит в следующем.

ЗАДАчА 2.1. Пусть $m \geqslant 0$, а $X$-компакт в $\mathbb{C}$. Каковы условия на $m$ и $X$, необходимые и достаточные для того, чтобы выполнялось равенство

$$
C^{m}(X) \cap S H\left(X^{\circ}\right)=\left.\left(C^{m}(\mathbb{C}) \cap S H(\mathbb{C})\right)\right|_{X} ?
$$

Дополнительный интерес представляет получение соответствующих оценок для норм найденных продолжений.

Как видно из $\S 1$, в настоящей работе мы ограничиваемся рассмотрением случаев, когда $X$ - замкнутая жорданова область в $\mathbb{C}$.

Аналогичный задаче 2.1 вопрос о Lip ${ }^{m}$-продолжении субгармонических фуункций обсуждается в $\S 4$. 


\section{§3. Доказательство теоремы 1.1}

Пусть $B\left(z_{0}, r\right)$ - открытьй круг в $\mathbb{C}$ с центром в точке $z_{0}$ и радиусом $r>0$. Через $c, c_{2}, c_{3}, \ldots$ будут обозначаться положительные константы, которые (в конечном счете) определяются только константой $c_{1}$ (см. (1.1)) и параметром $m$. При этом $c$ может меняться в разных соотношениях, а $c_{1}, c_{2}, \ldots$ фиксируются. Отметим, что до конца этого параграфа вьполнены условия и используются обозначения теоремы 1.1. Фиксируем некоторую точку $a$ в $D$ и число $R \in(0, \operatorname{diam} D)$ такие, что $D \subset B(a, R)$. Через $d(z)$ обозначим расстояние от точки $z$ до компакта $\bar{D}$.

Докажем сначала следующую лемму, сводящую теорему 1.1 при $m \in[2,3)$ к случаю $f \in C^{m}(\bar{D}) \cap H(D)$.

Лемма 3.1. Пусть $m \in[2,3), f \in C^{m}(\bar{D}) \cap S H(D)$. Тогда найдется функиия $F_{1} \in C^{m}(\mathbb{C}) \cap S H(\mathbb{C})$ такая, что:

a) $f-F_{1} \in H(D)$;

b) $\left\|F_{1}\right\|_{m, \bar{D}} \leqslant c\left\|\nabla^{2} f\right\|_{m-2, \bar{D}} \leqslant c\|f\|_{m, \bar{D}}$;

c) $\left\|\nabla F_{1}\right\|_{m-1} \leqslant c\left\|\nabla^{2} f\right\|_{m-2, \bar{D}} \leqslant c\|\nabla f\|_{m-1, \bar{D}}$.

ДокАзАтельСтво. Рассматривая вместо $f$ функцию $f_{a}^{*}(x)=f(x)-f(a)-$ $\left.(\nabla f)\right|_{a}(x-a)$ (отличающуюся от $f$ на линейную, т. е. гармоническую функцию), мы можем без ограничения обшности считать, что $f(a)=|(\nabla f)|_{a} \mid=0$, поскольку

$$
\left\|\nabla^{2} f\right\|_{m-2, \bar{D}}=\left\|\nabla^{2} f_{a}^{*}\right\|_{m-2, \bar{D}}, \quad\left\|\nabla f_{a}^{*}\right\|_{m-1, \bar{D}} \leqslant 2\|\nabla f\|_{m-1, \bar{D}} .
$$

Важно то, что при $f(a)=|(\nabla f)|_{a} \mid=0$ имеют место оценки

$$
\|f\|_{m, \bar{D}} \leqslant c\|\nabla f\|_{m-1, \bar{D}} \leqslant c^{2}\left\|\nabla^{2} f\right\|_{m-2, \bar{D}} .
$$

Рассмотрим сначала случаи $m \in(2,3)$. По теореме Уитни [2, гл. $6, \S 2.3$ и $\S 4.6]$ продолжим функцию $\Delta f \in C^{m-2}(\bar{D})$ до функции $h_{1} \in C^{m-2}(\mathbb{C})$ с условиями $h_{1} \geqslant 0$ всюду в $\mathbb{C}, \operatorname{supp} h_{1} \subset B(a, 2 R)_{\text {и }}$

$$
\left\|h_{1}\right\|_{m-2} \leqslant c\|\Delta f\|_{m-2, \bar{D}}
$$

где $\operatorname{supp} h$ - носитель (обобщенной) функции $h$. Пусть $\Phi(z)=(2 \pi)^{-1} \ln |z|-$ фундаментальное решение для уравнения Лапласа в $\mathbb{R}^{2}$. Положим $F_{1}=\Phi * h_{1}$, где через $*$ обозначена операция свертки. Проверим, что $F_{1}-$ искомая функция. Условие $F_{1} \in S H(\mathbb{C})$ и свойство а) очевидны. Определим

$$
\partial_{z}=\frac{\partial}{\partial z}=\frac{1}{2}\left(\frac{\partial}{\partial x_{1}}-i \frac{\partial}{\partial x_{2}}\right), \quad \partial_{\bar{z}}=\frac{\partial}{\partial \bar{z}}=\frac{1}{2}\left(\frac{\partial}{\partial x_{1}}+i \frac{\partial}{\partial x_{2}}\right) .
$$

Имеем

$$
\partial_{z} F_{1}=\left(\partial_{z} \Phi\right) * h_{1}=\frac{1}{4 \pi z} * h_{1} .
$$

Поскольку $F_{1}$ вещественна, справедливо равенство $\left|\nabla F_{1}(x)\right|=2\left|\partial_{z} F_{1}\right|$, так что требуемые оценки для $\left\|F_{1}\right\|_{\bar{D}}$ и $\left\|\nabla F_{1}\right\|$ непосредственно следуют из локальной интегрируемости (по мере Лебега в $\mathbb{R}^{2}$ ) логарифмического ядра $\Phi$ и ядра Коши $4 \partial_{z} \Phi$. Таким образом, ввиду (3.2) для завершения доказательства неравенств b) 
и с) остается оценить $C^{m-2}$-норму частных производных второго порядка функции $F_{1}$. Поскольку $\Delta F_{1}=h_{1}$ и $\partial_{z}^{2} F_{1}=\overline{\partial \frac{2}{z} F_{1}}$, то необходимо получить оценку для $\left\|\partial_{z}^{2} F_{1}\right\|_{m-2}$. Нетрудно показать, что (в обобшенном смысле) справедливо равенство

$$
\frac{\partial^{2} F_{1}}{\partial z^{2}}=-\frac{1}{4 \pi z^{2}} * h_{1}
$$

поэтому требуемая оценка

$$
\left\|\partial_{z}^{2} F_{1}\right\|_{m-2} \leqslant c\left\|h_{1}\right\|_{m-2}
$$

вытекает из хорошо известных свойств непрерывности оператора КальдеронаЗигмунда с ядром $1 / z^{2}$ (по мере Лебега в $\mathbb{C}$ ), действуюшего из пространства $B C_{0}^{\mu}(B(a, 2 R))$ (функций класса $B C^{\mu}$ с носителем в круге $\left.B(a, 2 R)\right)$ в пространство $B C^{\mu}$ при любом фиксированном $\mu \in(0,1)$ (в рассматриваемом случае $\mu=$ $m-2)$.

Пусть теперь $m=2$. По указанной вьше теореме Уитни продолжим функцию $f$ до функции $F_{0} \in B C^{2}(\mathbb{C})$ с условиями $\operatorname{supp} F_{0} \subset B(a, R)$ и $\left\|F_{0}\right\|_{2} \leqslant c\|f\|_{2, \bar{D}}$. Пусть $\Psi_{1}(x)=\max \left\{0,-\Delta F_{0}(x)\right\}$. Ясно, что $\Psi_{1}$ непрерывна и неотрицательна в $\mathbb{R}^{2}$, причем supp $\Psi_{1} \subset B(a, R) \backslash D$. Пусть $h_{1}(w)=0$ при $|w| \leqslant 1$, а при $|w|>1$ положим

$$
h_{1}(w)=\max _{\{z:|k(z)|=|w|\}} \Psi_{1}(z) .
$$

$\Phi$ ункция $h_{1}$ также неотрицательна и непрерывна в $\mathbb{R}^{2}$, имеет компактный носитель и радиально-симметрична: $h_{1}(w)=h_{1}(|w|)$. Имеем

$$
\left\|h_{1}\right\|=\left\|\Psi_{1}\right\| \leqslant\left\|\Delta F_{0}\right\| \leqslant c\|f\|_{2, \bar{D}}
$$

Найдем $H_{1} \in C^{2}\left(\mathbb{R}^{2}\right)$ с условиями $\Delta H_{1}=h_{1}$ и $H \equiv 0$ в $B_{1}$. Переходя к полярным координатам, легко убедиться, что (единственное) решение последней задачи имеет вид

$$
\begin{array}{ll}
H_{1}(w)=H_{1}(\rho)=0, & |w|=\rho \leqslant 1 \\
H_{1}(w)=H_{1}(\rho)=\int_{1}^{\rho} \frac{d t}{t} \int_{1}^{t} \tau h_{1}(\tau) d \tau, & |w|=\rho>1 .
\end{array}
$$

Дифференцируя последнее равенство по параметру $\rho$ и используя элементарные соотношения

$$
\left|\nabla H_{1}(w)\right|=\left|H_{1}^{\prime}(\rho)\right|, \quad\left|\nabla^{2} H_{1}(w)\right| \leqslant\left|H_{1}^{\prime \prime}(\rho)\right|+\frac{1}{\rho}\left|H_{1}^{\prime}(\rho)\right|,
$$

находим, что $H_{1} \in C^{2}(\mathbb{C}), H_{1}(w)=0$ при $|w| \leqslant 1$, причем

$$
\left\|\nabla H_{1}\right\| \leqslant c\left\|h_{1}\right\|, \quad\left\|\nabla^{2} H_{1}\right\| \leqslant c\left\|h_{1}\right\| .
$$


Пусть $g_{1}(z)=0$ при $z \in \bar{D}$ и $g_{1}(z)=H_{1}(k(z))$ при $z \in \mathbb{C} \backslash \bar{D}$. Из условия (1.1), вещественности функции $g_{1}$ и из соотношений (при $z \in \mathbb{C} \backslash \bar{D}$ )

$$
\begin{gathered}
\frac{\partial g_{1}(z)}{\partial z}=\left.\frac{\partial H_{1}}{\partial w}\right|_{k(z)} k^{\prime}(z), \\
\frac{\partial^{2} g_{1}(z)}{\partial z^{2}}=\left.\frac{\partial^{2} H_{1}}{\partial w^{2}}\right|_{k(z)}\left(k^{\prime}(z)\right)^{2}+\left.\frac{\partial H_{1}}{\partial w}\right|_{k(z)} k^{\prime \prime}(z), \\
\Delta g_{1}(z)=\left.\Delta H_{1}\right|_{k(z)}\left|k^{\prime}(z)\right|^{2}, \\
\left|k^{\prime \prime}(z)\right| \leqslant \frac{c_{1}}{d(z)},\left.\quad \frac{\partial H_{1}}{\partial w}\right|_{k(z)}=o(d(z)), \\
\left|\frac{\partial H_{1}}{\partial w}\right|_{k(z)}|\leqslant| H_{1}^{\prime}(|k(z)|) \mid \leqslant(|k(z)|-1)\left\|h_{1}\right\| \leqslant c_{1} d(z)\left\|h_{1}\right\|
\end{gathered}
$$

получаем, что $g_{1} \in C^{2}(\mathbb{C}) \cap S H(\mathbb{C}),\left\|\nabla g_{1}\right\| \leqslant c\left\|h_{1}\right\|$ и $\left\|\nabla^{2} g_{1}\right\| \leqslant c\left\|h_{1}\right\|$. Применяя оценки (3.3) и (3.1), получаем, что искомую функцию $F_{1}$ можно взять в виде $F_{0}+\left(c_{1}\right)^{2} g_{1}$. Лемма доказана.

Остальные этапы доказательства теоремы 1.1 одинаковы для всех $m \in(1,3)$.

Лемма 3.2. Пусть $m \in(1,3)$. Найдутся константы $c>0, c_{2}>0$ и функиия $F_{2} \in S H(\mathbb{C}) \cap \operatorname{Lip}^{m}(\mathbb{C}) \cap C^{\infty}(\mathbb{C} \backslash \partial D)$ такие, ито:

a) $F_{2}(z)=0$ npu $z \in \bar{D}$;

b) $\left\|\nabla F_{2}\right\|_{m-1} \leqslant c$;

c) $\left|\Delta F_{2}(z)\right| \geqslant c_{2}(d(z))^{m-2}$ nрu $z \in B(a, 2 R) \backslash \bar{D}$.

ДокАЗАТЕЛЬСтво. Рассмотрим функцию $G_{2}(w)=0$ при $|w| \leqslant 1, G_{2}(w)=$ $(\ln |w|)^{m}$ при $|w|>1$. Элементарно проверяется, что $G_{2}$ удовлетворяет всем условиям леммы в случае $D=B_{1}$. В частности, при $|w|>1$ имеем

$$
\begin{gathered}
\left|\nabla G_{2}(w)\right|=m(\ln |w|)^{m-1}|w|^{-1} \\
\left|\nabla^{2} G_{2}(w)\right| \leqslant c\left((\ln |w|)^{m-2}+(\ln |w|)^{m-1}\right)|w|^{-2} \\
\Delta G_{2}(w)=m(m-1)(|w|)^{-2}(\ln |w|)^{m-2}>0
\end{gathered}
$$

поэтому найдется $c_{3}>0$ такая, что

$$
\Delta G_{2}(w) \geqslant c_{3}(|w|-1)^{m-2}
$$

при $w \in k(B(a, 2 R) \backslash \bar{D})$.

Рассмотрим функцию $F_{2}(z)$, равную нулю при всех $z \in \bar{D}$ и равную $G_{2}(k(z))$ при $z \in \mathbb{C} \backslash \bar{D}$. При $z \in \mathbb{C} \backslash \bar{D}$ имеем

$$
\partial_{z} F_{2}=\overline{\partial_{\bar{z}} F_{2}}=\left.\partial_{w} G(w)\right|_{k(z)} k^{\prime}(z)=m(\ln |k(z)|)^{m-1}(2 k(z))^{-1} k^{\prime}(z),
$$

откуда легко видеть, что $F_{2} \in C^{1}(\mathbb{C}) \cap C^{\infty}(\mathbb{C} \backslash \partial D)$ и

$$
\left\|\nabla F_{2}\right\| \leqslant c=c\left(m, c_{1}\right) .
$$


Далее, при $z \in \mathbb{C} \backslash \bar{D}$ имеем

$$
\Delta F_{2}(z)=4 \partial_{z} \partial_{\bar{z}} F_{2}=\left.\Delta G_{2}(w)\right|_{k(z)}\left|k^{\prime}(z)\right|^{2} \geqslant 0 .
$$

Поскольку $F_{2} \in S H(\mathbb{C} \backslash \partial D) \cap C^{1}(\mathbb{C})$ и $\partial D$ спрямляема, можно показать (используя, например, обобщенную формулу Гаусса-Остроградского, как это сделано в конще доказательства теоремы 2.2 работы [1]), что $F_{2} \in S H(\mathbb{C})$. Свойство с) (с константой $c_{2}=c_{3} c_{1}^{-3}$ ) непосредственно вытекает из (3.6), (3.4) и (1.1). Остается проверить, что $F_{2} \in \operatorname{Lip}^{m}(\mathbb{C})$, и установить неравенство b). При $z \in \mathbb{C} \backslash \bar{D}$ имеем

$$
\partial_{z}^{2} F_{2}(z)=\overline{\partial_{\bar{z}}^{2} F_{2}(z)}=\left.\partial_{w}^{2} G_{2}(w)\right|_{k(z)}\left(k^{\prime}(z)\right)^{2}+\left.\partial_{w} G_{2}(w)\right|_{k(z)} k^{\prime \prime}(z),
$$

откуда нетрудно показать, что при $m \in(2,3)$ имеем $F_{2} \in C^{2}(\mathbb{C})$ (а при $m=2$ имеем $\left.F_{2} \in \operatorname{Lip}^{2}(\mathbb{C}) \cap C^{\infty}(\mathbb{C} \backslash \partial D)\right)$ и справедливы нужные равномерные оценки вторых частных производных функции $F_{2}$. В частности, случай $m=2$ в дальнейшем рассмотрении не нуждается.

Пусть $m=m_{0}+\mu$, где $m_{0}$ - целая часть $m$ и $\mu \in(0,1)$. Остается надлежащим образом оценить $\omega_{\mathbb{C}}^{m_{0}, \mu}\left(F_{2}, \infty\right)$. Мы обсудим только случай $m_{0}=1$, случай $m_{0}=2$ рассматривается аналогично ( $\omega_{\mathbb{C}}^{\mu}$ оценивается отдельно для $\Delta F_{2}$ и отдельно для каждого слагаемого в правой части формулы (3.7)).

Итак, пусть $m=1+\mu$. Возьмем произвольные $z_{1}$ и $z_{2}$ в $\mathbb{C},\left|z_{1}-z_{2}\right|=\delta>0$. Согласно (3.5) достаточно установить неравенство

$$
\left|\partial_{z} F_{2}\left(z_{2}\right)-\partial_{z} F_{2}\left(z_{1}\right)\right| \leqslant c \delta^{\mu} .
$$

Поскольку $F_{2} \in C^{1}(\mathbb{C})$ и $F_{2}=0$ в $\bar{D}$, достаточно рассмотреть случай, когда обе точки $z_{1}$ и $z_{2}$ лежат в $\mathbb{C} \backslash \bar{D}$. Пусть $d_{j}=d\left(z_{j}\right)$ и $w_{j}=k\left(z_{j}\right)$ при $j \in\{1,2\}$. Без ограничения общности предполагаем, что $d_{2} \geqslant d_{1}$. Согласно (1.1) имеем

$$
\left|w_{2}-w_{1}\right| \in\left[c_{1}^{-1} \delta, c_{1} \delta\right], \quad\left|w_{j}\right|-1 \in\left[c_{1}^{-1} d_{j}, c_{1} d_{j}\right]
$$

при $j \in\{1,2\}$.

Рассмотрим два случая. Пусть сначала $\delta \geqslant d_{2} / 2$, откуда $d_{1} \leqslant d_{2} \leqslant 2 \delta$. Ввиду (3.8) и упомянутых выше свойств функции $G_{2}$ имеем

$$
\begin{aligned}
& \left|\partial_{z} F_{2}\left(z_{2}\right)-\partial_{z} F_{2}\left(z_{1}\right)\right| \leqslant\left|\partial_{w} G\left(w_{2}\right)-\partial_{w} G\left(w_{1}\right)\right|\left|k^{\prime}\left(z_{2}\right)\right| \\
& \quad+\left|\partial_{w} G\left(w_{1}\right)\right|\left|k^{\prime}\left(z_{2}\right)-k^{\prime}\left(z_{1}\right)\right| \leqslant c\left|w_{1}-w_{2}\right|^{\mu} c_{1}+c d_{1}^{\mu} c_{1} \leqslant c\left(m, c_{1}\right) \delta^{\mu} .
\end{aligned}
$$

Пусть теперь $\delta<d_{2} / 2$. Поскольку в этом случае $\left|k^{\prime}\left(z_{2}\right)-k^{\prime}\left(z_{1}\right)\right| \leqslant c\left|z_{1}-z_{2}\right| / d_{2}$, мы окончательно получаем

$$
\begin{aligned}
& \left|\partial_{z} F_{2}\left(z_{2}\right)-\partial_{z} F_{2}\left(z_{1}\right)\right| \leqslant\left|\partial_{w} G\left(w_{2}\right)-\partial_{w} G\left(w_{1}\right)\right|\left|k^{\prime}\left(z_{2}\right)\right| \\
& \quad+\left|\partial_{w} G\left(w_{1}\right)\right|\left|k^{\prime}\left(z_{2}\right)-k^{\prime}\left(z_{1}\right)\right| \leqslant c \delta^{\mu}+c d_{1}^{\mu} \delta\left(d_{2}\right)^{-1} \leqslant c\left(m, c_{1}\right) \delta^{\mu} .
\end{aligned}
$$


Лемма 3.3. Пусть $f \in S H(D) \cap C^{m}(\bar{D})$ nри $m \in(1,2)$ или $f \in H(D) \cap$ $C^{m}(\bar{D})$ при $m \in[2,3)$. Найдутся константа $c>0$ и функиия $F_{3} \in S H(\mathbb{C}) \cap$ $\operatorname{Lip}^{m}(\mathbb{C}) \cap C^{m}(\mathbb{C} \backslash \partial D)$ такие, ито:

a) $F_{3}(z)=f(z)$ npu $z \in \bar{D}$;

b) $\left\|\nabla F_{3}\right\|_{m-1} \leqslant c\|\nabla f\|_{m-1, \bar{D}}$.

ДОКАЗАТЕЛЬСТВО. Без ограничения общности мы можем предположить, что $f(a)=0$, так что $\|f\|_{m, \bar{D}} \leqslant c\|\nabla f\|_{m-1, \bar{D}}$. Продолжим функцию $f$ (с множества $\bar{D})$ до функции $F_{0} \in B C^{m}(\mathbb{C})$ с носителем в круге $B(a, 2 R)$ и условиями

$$
\left\|F_{0}\right\|_{m} \leqslant c\|f\|_{m, \bar{D}}, \quad\left|\Delta F_{0}(z)\right| \leqslant c\|f\|_{m, \bar{D}}(d(z))^{m-2}
$$

Последняя оценка при $m \in(1,2)$ вытекает из $\left[2\right.$, гл. $6, \S 2.3$, оценка $\left.2.3 .2\left(\sigma^{\prime}\right)\right]$, а при $m \in[2,3)$ она непосредственно следует из определения нормы $\|\cdot\|_{m}$ и условия $\Delta F_{0}=0$ в $\bar{D}$. Из леммы 3.2 получаем, что найдется $c_{4}>0$ такая, что функция

$$
F_{3}=F_{0}+c_{4}\|f\|_{m, \bar{D}} F_{2}
$$

удовлетворяет всем требованиям леммы 3.3 .

Лемма 3.4. Пусть $f \in S H(D) \cap C^{m}(\bar{D})$ nрu $m \in(1,2)$ или $f \in H(D) \cap$ $C^{m}(\bar{D})$ при $m \in[2,3)$. Тогда для любого $\varepsilon \in(0,1)$ найдется функиия $F_{\varepsilon} \in$ $\mathrm{SH}(\mathbb{C}) \cap C^{m}(\mathbb{C})$ такая, что:

a) ecли $m \in(1,2), \operatorname{mo}\left(f-F_{\varepsilon}\right) \in S H(D) u \operatorname{supp} \Delta F_{\varepsilon} \cap D \subset \operatorname{supp} \Delta f ;$ ecлu $m \in[2,3)$, то $\left(f-F_{\varepsilon}\right) \in H(D)$ (в частности, $\left.F_{\varepsilon} \in H(D)\right)$;

b) $\left\|f-F_{\varepsilon}\right\|_{m, \bar{D}}<\varepsilon$;

c) $\left\|\nabla F_{\varepsilon}\right\|_{m-1} \leqslant c\|\nabla f\|_{m-1, \bar{D}}$.

ДокАЗАТЕЛЬСтво. Как и ранее, мы можем предположить, что $f(a)=0$ (откуда $\left.\|f\|_{m, \bar{D}} \leqslant c\|\nabla f\|_{m-1, \bar{D}}\right)$, и продолжить функцию $f$ до функции $F_{0} \in B C^{m}(\mathbb{C})$ с носителем в круге $B(a, 2 R)$ и условием $\left\|F_{0}\right\|_{m} \leqslant c\|f\|_{m, \bar{D}}$. Фиксируем произвольное $\varepsilon>0$.

Утверждается, что найдется функция $G_{\varepsilon} \in B C^{m}(\mathbb{C}) \cap S H(D)$ (если $m \in$ $[2,3)$, то $\left.G_{\varepsilon} \in B C^{m}(\mathbb{C}) \cap H(D)\right)$, гармоническая в некоторой окрестности $U=$ $U(\partial D)$ гранищы $\partial D$ области $D$ с условиями

$$
\left\|G_{\varepsilon}-F_{0}\right\|_{m}<\varepsilon, \quad\left(f-G_{\varepsilon}\right) \in S H(D), \quad \operatorname{supp} \Delta G_{\varepsilon} \cap D \subset \operatorname{supp} \Delta f .
$$

При $m \in[2,3)$ указанное утверждение (о возможности гармонической $C^{m}$-аппроксимации) непосредственно следует из теоремы О'Фаррелла-Вердеры (см. [3, теорема $\left.1^{\prime \prime}\right]$ и $[4$, теорема 1$\left.]\right)$. Пусть теперь $m \in(1,2)$. При $\delta \in(0, R / 4)$ рассмотрим стандартное $\delta$-разбиение единицы $\left\{B_{j}, \varphi_{j}\right\}_{j \in \mathbb{Z}^{2}}$ в $\mathbb{C}$ (здесь $j=\left(j_{1}, j_{2}\right)$, $\left.a_{j}=\left(\delta j_{1}, \delta j_{2}\right), B_{j}=B\left(a_{j}, \delta\right), \varphi_{j} \in C_{0}^{\infty}\left(B_{j}\right), \sum_{j \in \mathbb{Z}^{2}} \varphi_{j} \equiv 1,\left\|\nabla^{2} \varphi_{j}\right\| \leqslant c \delta^{-2}\right)$. Пусть $f_{j}=\Phi *\left(\varphi_{j} \Delta F_{0}\right)$ - соответствуюшие локализованные (по схеме Витушкина [5]) функции. Положим $J=\left\{j \in \mathbb{Z}^{2} \mid B_{j} \cap B(a, 2 R) \neq \varnothing\right\}, J_{1}=\left\{j \in \mathbb{Z}^{2} \mid B_{j} \cap\right.$ $\partial D \neq \varnothing\}$. Ввиду (1.1) для каждого $j \in J_{1}$ существует (замкнутый) круг $\bar{B}_{j}^{*}$ радиуса $\left(2 c_{1}\right)^{-2} \delta$, принадлежащий множеству $B\left(a_{j}, 2 \delta\right) \backslash \bar{D}$. Нетрудно показать 
(см. $\left[4\right.$, доказательство теоремы 2]), что найдутся функции $g_{j} \in C^{m}(\mathbb{C}) \cap H\left(\mathbb{C} \backslash \bar{B}_{j}^{*}\right)$, $j \in J_{1}$, такие, что

$$
\left\|\sum_{j \in J_{1}}\left(f_{j}-g_{j}\right)\right\|_{m}<c \omega_{\mathbb{C}}^{m-1}\left(F_{0}, \delta\right) .
$$

Поскольку $F_{0}=\sum_{j \in J} f_{j}$ и $\omega_{\mathbb{C}}^{m-1}\left(F_{0}, \delta\right) \rightarrow 0$ при $\delta \rightarrow 0$, то при достаточно малом $\delta$ искомую функцию $G_{\varepsilon}$ можно взять в виде

$$
G_{\varepsilon}=\sum_{j \in J \backslash J_{1}} f_{j}+\sum_{j \in J_{1}} g_{j} .
$$

Требуемое утвержсение доказано.

Продолжим доказательство леммы. Выберем $\delta_{1} \in(0,1)$ такое, что область $D_{1}$, ограниченная кривой $k^{-1}\left(\left\{w \in \mathbb{C}|| w \mid=1+\delta_{1}\right\}\right)$, содержится в множестве $D \cup U$. При этом $k_{1}(z)=k(z) /\left(1+\delta_{1}\right)$ - конформное отображение области $\overline{\mathbb{C}} \backslash \bar{D}_{1}$ на $\overline{\mathbb{C}} \backslash \bar{B}_{1}$, удовлетворяющее условию $c_{1}\left(D_{1}\right) \leqslant\left(1+\delta_{1}\right) c_{1} \leqslant 2 c_{1}$, где $c_{1}\left(D_{1}\right)$ - константа "билипшицевости" функции $k_{1}$ (т. е. константа в формуле, аналогичной $(1.1)$, для $\left.k_{1}\right)$. Пусть теперь $G_{3}$ - построенная в лемме 3.3 (вместо $F_{3}$ ) функция (для области $D_{1}$ вместо $D$ и функции $\left.G_{\varepsilon}\right|_{\bar{D}_{1}}$ вместо $\left.f\right)$. Так же, как и в $[6$, доказательство леммы 5.2$]$, остается сгладить функцию $G_{3}$. Фиксируем $\rho \in\left(0, \operatorname{dist}\left(\partial D, \partial D_{1}\right) / 3\right)$. Выберем $p a-$ диально-сим.метричную функцию

$$
\varphi_{1} \in C_{0}^{\infty}\left(B_{1}\right), \quad \varphi_{1} \geqslant 0, \quad \int_{B_{1}} \varphi_{1}\left(x_{1}, x_{2}\right) d x_{1} d x_{2}=1
$$

и положим $\varphi_{\rho}(z)=\rho^{-2} \varphi(z / \rho)$. Функция $F_{\varepsilon}$, равная $G_{\varepsilon}$ в $2 \rho$-окрестности области $D$ и равная $\varphi_{\rho} * G_{\varepsilon}$ вне $\rho$-окрестности области $D$, является искомой. Действительно, $\varphi_{\rho} * G_{\varepsilon} \in C^{m}(\mathbb{C})$, причем

$$
\left\|\nabla\left(\varphi_{\rho} * G_{\varepsilon}\right)\right\|_{m-1} \leqslant c\left\|\nabla G_{\varepsilon}\right\|_{m-1} \leqslant c^{2}\|\nabla f\|_{m-1, \bar{D}}
$$

$G_{\varepsilon} \in C^{m}\left(\bar{D}_{1}\right)$ и $\left\|\nabla G_{\varepsilon}\right\|_{m-1, \bar{D}_{1}} \leqslant c\|\nabla f\|_{m-1, \bar{D}}$. При этом функции $\varphi_{\rho} * G_{\varepsilon}$ и $G_{\varepsilon}$ совпадают на множестве $\left\{z \in D_{1} \backslash D \mid \rho<\operatorname{dist}(z, \partial D)<2 \rho\right\}$, что вытекает из теоремы о среднем для гармонической в области $D_{1} \backslash \bar{D}$ функции $G_{\varepsilon}$ и указанных вьше свойств функции $\varphi_{\rho}$. Лемма доказана.

Завершим доказательство теоремы 1.1. Ввиду леммы 3.1 мы можем считать, что при $m \in[2,3)$ имеем $f \in H(D) \cap C^{m}(\bar{D})$. Так же, как и в [6], нам остается устроить итерационный процесс. Применяя лемму 3.4 к функции $f$ и $\varepsilon=1 / 2$, найдем $F_{\varepsilon_{1}}$. Применим снова лемму 3.4 к функции $\left.\left(f-F_{1}\right)\right|_{D}$ и $\varepsilon=1 / 2^{2}$, в результате получая $F_{\varepsilon_{2}}$. По индукции находим функции $F_{\varepsilon_{3}}, \ldots, F_{\varepsilon_{n}}, \ldots$ такие, что

$$
\begin{gathered}
\left\|f-\left(F_{\varepsilon_{1}}+\cdots+F_{\varepsilon_{n}}\right)\right\|_{m, \bar{D}} \leqslant 2^{-n}\|\nabla f\|_{m-1, \bar{D}} \\
\left\|\nabla F_{\varepsilon_{n}}\right\|_{m-1} \leqslant c 2^{-n}\|\nabla f\|_{m-1, \bar{D}}
\end{gathered}
$$

Функция $F=\sum_{n=1}^{+\infty} F_{\varepsilon_{n}}$ определяет нужное продолжение. Теорема 1.1 доказана. 


\section{§4. Следствия}

В качестве первого следствия теоремы 1.1 мы получим “локализационную" теорему о $C^{m}$-субгармоническом продолжении с замкнутых жордановых областей.

ТЕОрема 4.1. Пусть $D$ - жсорданова область в $\mathbb{C}, m \in[1,3)$ u $f \in$ $C^{m}(\bar{D}) \cap S H(D)$. Предположим, что для любого $a \in \partial D$ найдутся окрестность $U_{a}$ точки а в $\mathbb{C}$ и функиия $F_{a} \in C^{m}\left(U_{a}\right) \cap S H\left(U_{a}\right)$ такие, ито $F_{a}=f$ в $D \cap U_{a}$. Тогда найдется $F \in C^{m}(\mathbb{C}) \cap S H(\mathbb{C})$ с условием $F=f$ в $\bar{D}$.

Случай $m=1$ этой теоремы был ранее получен в [1, теорема 2.5]. Хотя доказательство теоремы 4.1 в основном следует доказательству [1, теорема 2.5], мы приведем его для полноты изложения ввиду его краткости.

ДоКАЗАТЕЛЬСТВо. Поскольку множество

$$
E=\left\{e \in \partial D \mid \exists b_{e} \notin \bar{D}: \overline{B\left(b_{e},\left|b_{e}-e\right|\right)} \cap \bar{D}=\{e\}\right\}
$$

всюду плотно на $\partial D$, из компактности множества $\partial D$ следует, что найдутся конечные подмножества $A_{J}=\left\{a_{1}, \ldots, a_{J}\right\}$ в $\partial D$ и $E_{J}=\left\{e_{1}, \ldots, e_{J}\right\}$ в $E$, а также окрестности $U_{j}:=U_{a_{j}}$ точек $a_{j}$ со следующими свойствами: $\partial D \subset \bigcup_{j=1}^{J} U_{j}$; при каждом $j$ множество $U_{j}$ и соответствующая функция $F_{j}:=F_{a_{j}}$ удовлетворяют условиям теоремы 4.1 , причем $\gamma_{j}=\partial D \cap U_{j}$ - открытая жорданова дуга; точки $a_{j}$ занумерованы в последовательном порядке на $\partial D$ (полагаем $a_{J+1}=a_{1}$, $\left.U_{J+1}=U_{1}, F_{J+1}=F_{1}\right)$, окрестности $U_{j}$ (а также соответствуюшие дуги $\left.\gamma_{j}\right)$ пересекаются только попарно (последовательно) и $e_{j} \in \gamma_{j} \cap \gamma_{j+1}$. Круги $B^{j}=$ $B\left(b_{e_{j}},\left|b_{e_{j}}-e_{j}\right|\right), j=1, \ldots, J$, нужны для “разделения" несогласованных в $\left(U_{j} \cap\right.$ $\left.U_{j+1}\right) \backslash D$ функций $F_{j}$ и $F_{j+1}$.

Таким образом, найдется жорданова окрестность $\Omega$ множества $\bar{D}$ такая, что $f$ продолжается до функции $F_{J} \in C^{m}\left(\bar{\Omega}_{J}\right) \cap S H\left(\Omega_{J}\right)$, где

$$
\Omega_{J}=\Omega \backslash\left(\bar{B}^{1} \cup \cdots \cup \bar{B}^{J}\right) .
$$

Применяя теорему 1.1 в достаточно малых $B$-областях $G_{j}, 1 \leqslant j \leqslant J$, таких, что $G_{j} \subset \Omega_{J}$ и $G_{j} \cup \bar{B}^{j}$ содержит некоторую окрестность точки $e_{j}$, можно продолжить $F_{J}$ до $C^{m}$-субгармонической функции в некоторую окрестность множества $E_{J}$. Таким образом, в конечном счете $f$ надлежащим образом продолжается в некоторую окрестность множества $\bar{D}$. Для нахождения искомой функции $F$ остается применить теорему 1.1 к полученному продолжению и некоторой достаточно малой $B$-окрестности множества $\bar{D}$.

Следующее утверждение непосредственно вытекает из теоремы 4.1.

СЛЕДСТВИЕ 4.2. Пусть $D$ - произвольная жсорданова область в $\mathbb{C}, m \in$ $[1,3)$. Предположим, что для любой точки $a \in \partial D$ существует круг $B\left(a, r_{a}\right)$, $r_{a}>0$, такой, что для всякой функиии $f \in C^{m}(\bar{D}) \cap S H(D)$ найдется функиия $F_{a} \in C^{m}\left(\bar{D}_{a}\right) \cap S H\left(D_{a}\right), D_{a}=D \cup B\left(a, r_{a}\right)$, с условием $F_{a}=f$ в $\bar{D}$. Тогда для любой $f \in C^{m}(\bar{D}) \cap S H(D)$ найдется $F \in C^{m}(\mathbb{C}) \cap S H(\mathbb{C})$ с условием $F=f$ в $\bar{D}$. Если, кроме того, каждая функиия $F_{a}$ может быть выбрана с оченкой 
$\left\|\nabla F_{a}\right\|_{m-1, \bar{D}_{a}} \leqslant c(D, a)\|\nabla f\|_{m-1, \bar{D}}$, то указанная выше функция $F$ мохет быть выбрана с дополнительным условием $\|\nabla F\|_{m-1} \leqslant c(D)\|\nabla f\|_{m-1, \bar{D}}$.

Переформулируем теорему 1.1 в терминах продолжения соответствующей мерыг Рисса $\sigma=\Delta f$ функции $f$ из $D$ в $\mathbb{C}$.

СЛЕДСТВИЕ 4.3. Пусть $D$ является В-областью, $\Omega$ - некоторая окрестность множества $\bar{D}$ и $m \in(1,3)$. Найдется константа с $=c(D, \Omega, m)>0$ со следующими свойствами. Пусть $\sigma$-неотрицательная конечная борелевская мера в $D$ и $h$-гармоническая функиия в $D$ такие, что функиия

$$
\Phi * \sigma(x)+h(x)=\int_{D} \Phi(x-y) d \sigma(y)+h(x)
$$

продолжается (из D) до функции класса $C^{m}(\bar{D})$. Тогда найдется неотрицательная конечная борелевская мера $\sigma_{1}$ в $\Omega \backslash \bar{D}$ такая, что $\Phi *\left(\sigma+\sigma_{1}\right) \in C^{m}(\mathbb{C})$, причем

$$
\left\|\nabla \Phi *\left(\sigma+\sigma_{1}\right)\right\|_{m-1} \leqslant c\|\nabla(\Phi * \sigma+h)\|_{m-1, \bar{D}} .
$$

ДокАЗАТЕльство. Пусть $f=\Phi * \sigma+h$ (функция, определенная на $\bar{D}$ класса $\left.C^{m}(\bar{D})\right)$. Отметим, что мера $\sigma$ есть обобщенный лапласиан функции $f$ в $D$, причем согласно (обобшенной) формуле Гаусса-Остроградского имеем

$$
\int_{\partial D} \frac{\partial f}{\partial \nu} d \ell=\sigma(D)
$$

Здесь $\nu$ - единичная внешняя нормаль к $\partial D$ (сушествуюшая почти всюду относительно длины на $\partial D)$, а $d \ell$ - дифференциал длины на $\partial D$. Так что условие конечности меры $\sigma$ в $D$ необходимо. Продолжим функцию $f$ по теореме 1.1 до функции $F$. Фиксируем какую-либо функцию $\varphi \in C_{0}^{\infty}(\Omega)$ с условием $\varphi=1$ на $D$. Положим $F_{2}=\Phi *(\varphi \Delta F)$. Хорошо известно [4], что

$$
\left\|\nabla F_{2}\right\|_{m-1} \leqslant c(m, \varphi)\|\nabla F\|_{m-1} .
$$

Поскольку $F$ субгармонична в $\mathbb{C}$, ее мера Рисса $\Delta F$ есть неотрицательная локально конечная борелевская мера в $\mathbb{C}$, причем из упомянутой выше формулы Гаусса-Остроградского следует, что $\Delta F(\partial D)=0$. Искомая мера $\sigma_{1}$ имеет вид $\sigma_{1}=\varphi \Delta F-\sigma$.

Отметим, что следствие 4.3 справедливо и при $m=1$ для областей типа Дини-Ляпунова (как аналогичное следствие [1, теорема 2.2]).

Обсудим теперь аналоги задачи 2.1 и теоремы 1.1 для $\mathrm{Lip}^{m}$-продолжений субгармонических функций.

ТЕОрема 4.4. Пусть $D$ является $B$-областью, $m \in(1,3]$. Тогда найдется константа $c=c(D, m)>0$ такая, что для любой функции $f \in \operatorname{Lip}^{m}(\bar{D}) \cap$ $S H(D)$ существует $F \in \operatorname{Lip}^{m}(\mathbb{C}) \cap S H(\mathbb{C})$ с условиями $\left.F\right|_{\bar{D}}=f u$

$$
\|\nabla F\|_{m-1, \mathbb{C}} \leqslant c\|\nabla f\|_{m-1, \bar{D}} .
$$

При $m=1$ аналогичное утверждение справедливо для областей типа Дини-Ляпунова. 
ДокАЗАТЕЛЬСтво. Случай $m \in(1,2)$ непосредственно следует из леммы 3.2 и леммы 3.3 (ее аналога с заменой условия $f \in \mathrm{C}^{m}(\bar{D})$ на $\left.f \in \operatorname{Lip}^{m}(\bar{D})\right)$. При $m \in$ $[2,3]$ необходимо дополнительно воспользоваться следующим аналогом леммы 3.1 .

Лемма 4.5. Пусть $m \in[2,3], \quad f \in \operatorname{Lip}^{m}(\bar{D}) \cap S H(D)$. Тогда найдется функиия $F_{1} \in \operatorname{Lip}^{m}(\mathbb{C}) \cap S H(\mathbb{C})$ такая, что:

a) $f-F_{1} \in H(D)$;

b) $\left\|F_{1}\right\|_{m, \bar{D}} \leqslant c\left\|\nabla^{2} f\right\|_{m-2, \bar{D}} \leqslant c\|f\|_{m, \bar{D}}$;

c) $\left\|\nabla F_{1}\right\|_{m-1} \leqslant c\left\|\nabla^{2} f\right\|_{m-2, \bar{D}} \leqslant c\|\nabla f\|_{m-1, \bar{D}}$.

Чтобы установить справедливость леммы 4.5, дословно повторим доказательство леммы 3.1 с учетом непрерывности оператора Кальдерона-Зигмунда с ядром $1 / z^{2}$ (по мере Лебега в $\mathbb{C}$ ), действуюшего из пространства $\operatorname{Lip}_{0}^{\mu}(B(a, 2 R))$ (функций класса $\operatorname{Lip}^{\mu}(\mathbb{C})$ с носителем в круге $\left.B(a, 2 R)\right)$ в пространство $B \operatorname{Lip}^{\mu}(\mathbb{C})$ при (любом фиксированном) $\mu \in(0,1)(\mu=m-2)$. При $m=2$ и $m=3$ повторяется то же доказательство, что и в лемме 3.1 для случая $m=2$, только учитывается, что $\Psi_{1}$ и $h_{1}$ здесь будут принадлежать классу $L_{\infty}(\mathbb{C})($ при $m=2)$ или $\operatorname{Lip}^{1}(\mathbb{C})$ (при $m=3$ ).

В случае $m=1$ повторяется доказательство теоремы 2.2 работы [1] с некоторыми упрошениями (как, например, в настояшем доказательстве отпала необходимость в использовании леммы 3.4 и последуюших итераций в завершении доказательства теоремы 1.1).

СЛЕДСТВИЕ 4.6. Аналоги теоремы 4.1 и следствия 4.2 справедливы для Lip $^{m}$-продолэсений при $m \in[1,3]$.

В завершение работы обсудим вкратце задачу о $C^{m}$-субгармоническом продолжении (аналог задачи 2.1) в $\mathbb{R}^{N}$ (всюду далее $N \in\{3,4, \ldots\}$ фиксировано). Данные ранее (в $\S 1$ и $\S 2)$ определения пространств $S H(\Omega), H(\Omega)$, а также пространств $B C^{m}(\Omega), B \operatorname{Lip}^{m}(\Omega), C^{m}(\Omega), \operatorname{Lip}^{m}(\Omega), C^{m}(X), \operatorname{Lip}^{m}(X)$ дословно переносятся на открытые множества $\Omega$ и компакты $X$ в $\mathbb{R}^{N}$. Предложение 1.2 остается верным и для шаров в $\mathbb{R}^{N}$ (доказательство повторяется практически дословно). Однако, ввиду того, что всякое конформное отображение в (какой-либо области) $\mathbb{R}^{N}$ сводится к композициям подобия (сдвиг, гомотетия, ортогональное преобразование) и инверсии [7], аналоги теорем 1.1 и 4.4 в $N$-мерном случае удалось получить только для шаров. Соответственно, не удается доказать и аналог теоремы 4.1. Как уже отмечалось, при $m=1$ следующая теорема доказана в [6].

TeOpema 4.7. Пусть $B-$ uap в $\mathbb{R}^{N}, \quad m \in(1,3) u f \in \mathrm{C}^{m}(\bar{B}) \cap S H(B)$ (соответственно, $m \in(1,3]$ u $\left.f \in \operatorname{Lip}^{m}(\bar{B}) \cap S H(B)\right)$. Тогда найдется $F \in B C^{m}\left(\mathbb{R}^{N}\right) \cap S H\left(\mathbb{R}^{N}\right)$ (соответственно, $\left.F \in B \operatorname{Lip}^{m}\left(\mathbb{R}^{N}\right) \cap S H\left(\mathbb{R}^{N}\right)\right)$ с условиями $\left.F\right|_{\bar{B}}=f u$

$$
\|F\|_{m, \mathbb{R}^{N}} \leqslant c\|f\|_{m, \bar{B}}
$$

где $c \in(0,+\infty)$ зависит только от $N$ u $m$.

ДОКАЗАТЕЛЬСТВО. Мы можем считать, что $B=\{|x|<1\}$ - единичный шар. Пусть $\Phi_{N}(x)=-c_{N}|x|^{-N+2}$ - фундаментальное решение для оператора Лапласа в $\mathbb{R}^{N}$ ( $c_{N}>0$ - подходящая константа). Вторые (обобщенные) частные производные функции $\Phi_{N}$ являются ядрами типа Кальдерона-Зигмунда в $\mathbb{R}^{N}$ (см. $[2$, 
гл. $3, \S 1])$, поэтому доказательство леммы 3.1 для шаров в $\mathbb{R}^{N}$ проходит практически по той же схеме, как и в $\mathbb{R}^{2}$ (рассуждения, связанные с конформными отображениями, опускаются). В лемме 3.2 в качестве $F_{2}$ (или $G_{2}$ ) следует взять функцию $F_{2}(x)=\left(1-|x|^{2-N}\right)^{m}$ при $|x|>1, F_{2}(x)=0$ при $|x| \leqslant 1$. Изменения в доказательствах лемм 3.3 и 3.4 в особых комментариях не нуждаются. Оценка (4.2) (при $N=2$ она не верна) выглядит естественнее оценки (1.2). Дело здесь в том, что $\Phi_{N}$ (в отличие от $\left.\Phi_{2}=\Phi\right)$ ограничена в окрестности $\infty$ (в частности, функцию $f_{a}^{*}$ вместо $f$ в доказательствах лемм $3.1,3.3$ и 3.4 можно не рассматривать).

\section{Список литературы}

1. Мельников М. С., Парамонов П. В. $C^{1}$-продолжение субгармонических функций с замкнутых жордановых областей в $\mathbb{R}^{2}$ // Изв. РАН. Сер. матем. 2004. Т. 68. № 6. С. 105-118.

2. Стейн И. Сингулярные интегралы и дифференциальные свойства функций. М.: Мир, 1973.

3. O'Farrell A. G. Rational approximation in Lipschitz norms. II // Proc. Royal. Irish. Acad. 1979. V. 79A. № 11. P. 103-114.

4. Verdera J. $C^{m}$-approximation by solutions of elliptic equations, and Calderón-Zygmund operators // Duke Math. J. 1987. V. 55. № 1. P. 157-187.

5. Витушкин А.Г. Аналитическая емкость множеств в задачах теории приближений // УМН. 1967. Т. 22. №6. С. 141-199.

6. Вердера Дж., Мельников М. С., Парамонов П. В. $C^{1}$-аппроксимация и продолжение субгармонических функций // Матем. сб. 2001. Т. 192. № 4. С. 37-58.

7. Решетняк Ю.Г. Теорема Лиувилля о конформных отображениях при минимальных предположениях регулярности // Сиб. матем. жур. 1967. Т. 8. №4. С. 835-840.

МГУ им. М.В. Ломоносова

E-mail: petr@paramonov.msk.ru
Поступило в редакцию 23.05.2005 